Short report

\title{
EMG and histochemical findings in neurogenic atrophy with electrode localisation
}

\author{
JH PETAJAN, DJ THURMAN \\ From the Department of Neurology, University of Utah College of Medicine and \\ Veterans Administration Medical Center, Salt Lake City, Utah, USA
}

SUMmARY A new technique permitting electromyographic electrode localisation during open muscle biopsy is described. Using this technique recordings were made from biceps brachii from 22 normal subjects and 19 patients with neurogenic atrophy. Motor unit potential duration and onset interspike interval were the best predictors of histochemical evidence for atrophy. Of patients with neurogenic atrophy, polyphasic potentials and increased rise time of the major spike were found in 18 and 10 cases respectively. Abnormal motor unit potentials were always associated with findings of atrophy. However, normal duration, amplitude, and recruitment intervals occurred in $36 \%$, $58 \%$ and $50 \%$ respectively of subjects with neurogenic atrophy in the region of the recording.

The extent to which electromyographic (EMG) findings reflect pathologic changes in skeletal muscle as determined by histochemical techniques has been deduced primarily from circumstantial and post hoc evidence. A few studies of muscle biopsies following needle electrode recordings have yielded information concerning frequency control in relation to muscle fibre type. ${ }^{1}$ Single fibre electromyography has disclosed increased fibre density associated with reinnervation, particularly for type I fibres. ${ }^{2}$ Such studies are made difficult, however, because muscular pathology is highly variable within the biopsy cross section itself. We have sought to obtain a more adequate view of the relationship between histological changes and EMG findings by developing a procedure to identify the recording electrode site within the muscle after open biopsy. Patients with neurogenic atrophy, most of whom had amyotrophic lateral sclerosis, are the first to be reported using this technique.

\section{EMG recording procedure}

A Teflon ${ }^{(R)}$-coated 26 gauge monopolar recording electrode was prepared by applying to the tip an emulsion

Address for reprint requests: Prof Jack H Petajan, Department of Neurology, University of Utah College of Medicine, 50 North Medical Drive, Salt Lake City, Utah 84132, USA.

Accepted 16 June 1981 of carbon black, gum tragacanth and gelatin in distilled water. Several coats were applied and allowed to dry in air. A polyethylene No 10 tube was fixed to the electrode prior to coating, with about $2 \mathrm{~mm}$ of the electrode tip remaining exposed. At biopsy the electrode was inserted vertically into the muscle prior to injection of a local anaesthetic into muscle surrounding the biopsy site. The polyethylene tube sleeve prevented the electrode from sinking too deeply within the muscle. A small black spot was left at the origin of the needle track. Trials in vitro confirmed that the emulsion cast was left behind when the electrode was removed. The emulsion marked the area of recording and areas of muscle fibre damage caused by the electrode.

Motor unit potentials (MUPs) and spontaneous activity became recordable when the emulsion dissolved. A recording of MUPs was made for one minute at minimal effort and for 30 seconds upon recruitment of a second or third unit. Small adjustments in the depth of insertion were made, so that maximal amplitude and minimal duration of action potentials in the region of the recording tip were obtained. Potentials were amplified by a 3A9 Tektronix pre-amplifier at a sensitivity of 100-200 $\mu \mathrm{V} / \mathrm{cm}$ with a frequency range of $0 \cdot 1 \mathrm{~Hz}$ to $20 \mathrm{KHz}$ and recorded on an FM tape recorder. Electrode impedance was checked prior to preparation of the electrode and considered acceptable between 4 and $8 \mathrm{k} \Omega$. A 100 or 200 $\mu \mathrm{V} 10 \mathrm{~Hz}$ square pulse was recorded as calibration signal. Wave form characteristics were then measured on a storage oscilloscope. Duration and amplitude of 20 potentials were averaged. Measurements were not made until the variability of amplitude and duration was less than $10 \%$ at a $10 \mathrm{~ms} / \mathrm{cm}$ sweep speed. The interspike 
interval at onset (lower limiting) frequency was determined by measuring 10 successive intervals during a stable period of firing. ${ }^{3}$ Recruitment interval was defined as that interspike interval just preceding recruitment of a second motor unit action potential. ${ }^{3}$ Three to four instances of recruitment were measured in this manner.

\section{Histochemical preparations}

After recording, a biopsy of the bicepts brachii muscle was obtained. Frozen sections $10 \mu$ in thickness were stained with modified Gomori trichrome, haematoxylin and eosin, ATPase (pH 9.4), and NADH diaphorase. ${ }^{*}$ The muscle was sectioned up to and including the surface emulsion marker. Photomicrographs were made and lesser fibre diameters were measured as the distance between the two closest parallel lines laid tangential to opposite boundaries of the muscle fibre. Lesser diameter in relation to muscle fibre type as determined by the ATP-ase stain (pH 9.4) was recorded. Sections were inspected for uniformity of involvement by the apparent disease process. One hundred to 300 fibres were measured about the electrode tip estimated from the needle track. Where the needle track could not be defined $(25 \%$ of cases) fibres were measured and counted in regions most representative of the histochemical characteristics of the disease process.

\section{Results}

HISTOCHEMICAL FEATURES AND ELECTRODE LOCALISATION

Neurogenic atrophy was classified into three groups: Stage I-single fibre atrophy; Stage II-fibre type grouping. more than 15 fibres of one type being contiguous within a circle not containing a fibre of opposite types; Stage III-presence of grouped atrophy. Neurogenic atrophy was staged according to the most severe degree of involvement. Results for normal subjects and those with neurogenic atrophy are summarised in the table. Nineteen patients with neurogenic atrophy were studied. Twelve of these had amyotrophic lateral sclerosis (ALS), three spinal atrophy, one poliomyelitis with progression of weakness, and two polyneuropathy.
Stage I atrophy was present in none of the biopsies examined. Comparing the two groups, the mean and variance of fibre diameter for both types I and II fibres were higher in subjects with neurogenic atrophy owing to the presence of both hypertrophic and severely atrophic fibres. In specimens with stage III atrophy, an effort was made to measure fibre diameters which would reflect most accurately the overall alteration in fibre diameter.

Muscle fibres damaged by the electrode were infrequently seen and were always labelled by the emulsion. A fragment of emulsion indicating the recording site was usually seen in the section (fig). Electrode localisation was successful in $75 \%$ of cases.

MUP CHARACTERISTICS ASSOCIATED WITH NEUROGENIC ATROPHY

Abnormal total motor unit action potential duration and onset interval were the most sensitive indicators of stages II and III neurogenic atrophy (table). In ten cases, it was possible to ascertain a major spike and its rise time. In nine of these, prolonged rise time was associated with increased MUP duration. Increased rise time ( $>400 \mu \mathrm{s}$ ) was associated with "ragged" MUPs and recording from a large field of fibres of single type. In the remaining cases, marked polyphasia prevented definition of a single major spike.

Amplitude and recruitment intervals were less sensitive indicators. Eleven of nineteen subjects had a peak-to-peak amplitude of less than $2000 \mu \mathrm{V}$. Duration was normal (less than $18 \mathrm{~ms}$ and within two standard deviations of the mean of normal data) in seven of 19 biceps brachii with stage II or III neurogenic atrophy. Similarly, nine of 18 subjects with neurogenic atrophy had recruitment intervals less than or equal to $60 \mathrm{~ms} ; 50 \%$ had normal recruitment intervals. Seventeen of 19 subjects had polyphasic potentials, defined as a potential with greater than five phases or "ragged" potentials.

A plot of MUP duration versus coefficient of variation of diameter (standard deviation/mean $\times$

Table Biceps brachii muscle

\begin{tabular}{|c|c|c|c|c|c|c|}
\hline & Amplitude & Duration & Onset interial & $\begin{array}{l}\text { Recruitment } \\
\text { interval }\end{array}$ & $\begin{array}{l}\text { Diameter } \\
\text { fibre type I }\end{array}$ & $\begin{array}{l}\text { Diameter } \\
\text { fibre type II }\end{array}$ \\
\hline \multicolumn{7}{|c|}{ Normal } \\
\hline $\mathrm{N}$ & 22 & 22 & 20 & 21 & 22 & 22 \\
\hline Mean & $681: 2 \mathrm{~V}$ & $10 \mathrm{~ms}$ & $130 \mathrm{~ms}$ & $96 \mathrm{~ms}$ & $51: \mathrm{km}$ & $47 ! \mathrm{m}$ \\
\hline SD & $458: 2 \mathrm{~V}$ & $4 \mathrm{~ms}$ & $34 \mathrm{~ms}$ & $18 \mathrm{~ms}$ & $10 ! \mathrm{mm}$ & $11: \mathrm{Lm}$ \\
\hline \multicolumn{7}{|c|}{ Neurogenic atrophy } \\
\hline$N$ & 24 & 24 & 18 & 18 & 18 & 18 \\
\hline Mean & $1855 ! 2 \mathrm{~V}$ & $19 \mathrm{~ms}$ & $110 \mathrm{~ms}$ & $64 \mathrm{~ms}$ & $54 \mathrm{em}$ & $56 ! \mathrm{m}$ \\
\hline SD & $2006: \mathrm{V}$ & $9 \mathrm{~ms}$ & $34 \mathrm{~ms}$ & $22 \mathrm{~ms}$ & $12 \mathrm{~km}$ & $22: \mathrm{mm}$ \\
\hline \multicolumn{7}{|c|}{$\begin{array}{l}\text { Differences in } \\
\text { means significant }\end{array}$} \\
\hline at: $p$ & $<0.01$ & $<0.001$ & $<0.001$ & $<0.01$ & $<0.02$ & $<0.01$ \\
\hline
\end{tabular}




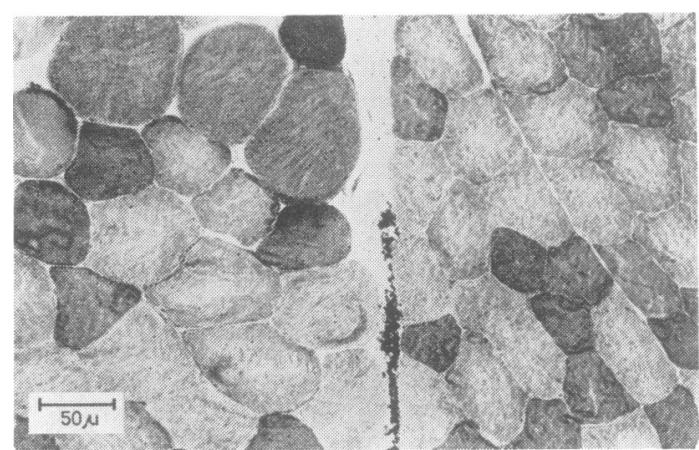

$1 \mathrm{a}$

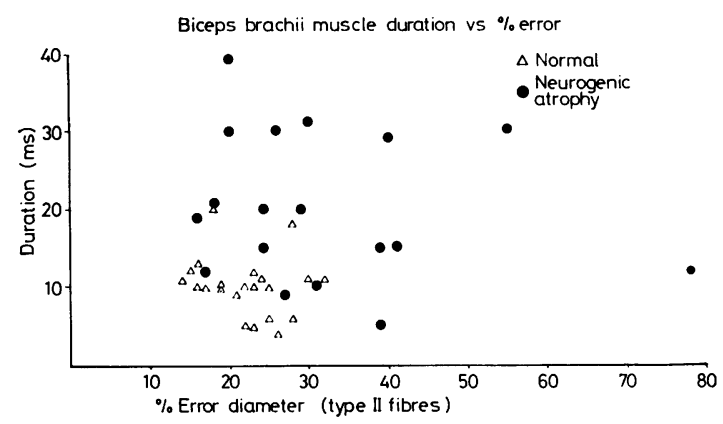

$1 b$

Fig 1 (a) A cross section of biceps brachii muscle from normal subject. $N A D H$ diaphorase stain; shows fragments of the India ink cast which localises the electrode recording tip. There is no muscle fibre damage. (b) Coefficient of variation of muscle fibre diameter for type II fibres is plotted against MUP duration for normal subjects and those with neurogenic atrophy.

100) reveals that increased duration can be present when the coefficient of variation is normal. Long durations were associated with higher mean diameters. No influence of fibre type upon these relationships was found. A trend toward normal duration was found as the coefficient of variation of fibre diameter increased, a finding characteristic of stage III atrophy.

\section{Discussion}

Increased total duration, as well as decreased onset intervals were found to be the most sensitive specific indicators of the most prominent histochemical findings, that is type grouping. In addition, increased rise time and "ragged" potentials consistently predicted stage II atrophy. Reduced rise time of the major spike has been shown to be a consistent characteristic of "myopathy". 5 However, MUPs can be normal when recorded from histochemically proven areas of stage II or III neurogenic atrophy; and the data suggest that, as the degree of fibre atrophy becomes more severe, duration and amplitude move toward normal.

Fibre diameter was often normal in the presence of increased total duration, rise time of major spike, polyphasia, and abnormal onset and recruitment intervals. Such changes probably result from spatial rearrangement of the motor unit and support the concept of collateral innervation. ${ }^{6}$ In the extreme case, a large "ragged" potential is associated with a large field of "type grouping". ${ }^{7} 8$ The spatial reorganisation of the unit results in greater temporal dispersion of individual fibre action potentials within the motor unit. ${ }^{9}$

An irregular loss of fibres within the reinnervated motor unit, ${ }^{10}$ may explain the presence of normal or even simple biphasic, low amplitude, short duration potentials. ${ }^{11}$ Muscle fibre density would be expected to decrease from the high levels found following reinnervation. ${ }^{12}$

In summary, the findings support the traditional view $^{13}$ that long duration, high amplitude, polyphasic MUPs with increased rise time of major spikes and decreased onset and recruitment intervals are associated with findings of stage II or III neurogenic atrophy. Fibre type grouping within a motor unit most likely accounts for these changes. However, normal MUPs or even potentials of short duration can be associated with stage II or III neurogenic atrophy. At the time of biopsy the EMG can be recorded from the biopsied region and not interfere with histochemical diagnosis.

We acknowledge the technical assistance of Del $\mathrm{McNeil}$ and Nina Beglarian.

\section{References}

1 Warmolts JR, Engel WK. Open biopsy electromyography I. Correlation of motor unit behaviour with histochemical muscle fiber type in human limb muscle. Arch Neurol 1972;27:512-7.

${ }^{2}$ Schwartz MS, Moosa A, Dubowitz V. Correlations of single fibre EMG and muscle histochemistry using an open biopsy recording technique. J Neurol Sci 1977; $31: 369-78$

${ }^{3}$ Petajan JH, Philip BA. Frequency control of motor unit action potentials. Electroencephalogr Clin Neurophysiol 1969;27:66-72.

${ }^{4}$ Dubowitz V, Brooke MH, Neville HE. Muscle biopsy: a modern approach. Histological and Histochemical Stains and Reactions 1973; Chapter 2:20-30.

${ }^{5}$ Simons AJR. Measurement of rise times of averaged muscle action potential in normal subjects and patients with myopathy. $J$ Neurol Neurosurg 
Psychiatry 1980;43:56-62.

${ }^{6}$ Buchthal F, Guld C, Rosenfalck P. Innervation zone and propagation velocity in human muscle. Acta Physiol (Scand) 1955;35:174-90.

' Edds MV. Collateral nerve regeneration. Quart Rev Biol 1953;28:260-76.

${ }^{8}$ Dubowitz V. Pathology of experimentally reinnervated skeletal muscle. J Neurol Neurosurg Psychiatry 1967;30:99-110.

${ }^{9}$ Karpati G, Engel WK. "Type grouping" in skeletal muscles after experimental reinnervation. Neurology (Minneap) 1969;18:447-55.

${ }^{10}$ Engel WK, Warmolts JR. The motor units diseases affecting it in toto or in portio. In: Desmedt JE, ed.
New Developments in Electromyography and Clinical Neurophysiology. Basel: Karger, 1973; Volume 1:141-77.

11 Warmolts JR, Mendell JR. Open biopsy electromyography. Direct correlation of a pattern of excessively recruited, pathologically small motor unit potentials with histologic evidence of neuropathy. Arch Neurol 1979;36:406-9.

${ }^{12}$ Schwartz MS, Stalberg E, Schiller HH, Thiele B. The reinnervated motor unit in man-a single fibre EMG electrode investigation. $J$ Neurol Sci 1976;27:303-12.

${ }^{13}$ Buchthal F, Pinelli P. Action potentials in muscular atrophy of neurogenic origin. Neurology (Minneap) 1953;3:591-603. 